Vol. 42 (1990) [487-497]

\title{
REGULARITY OF LOCALLY CONVEX SURFACES
}

\author{
Friedmar Schulz
}

Interior estimates are derived for the $C^{2, \mu}$-Hölder norm of the radius vector $X \in$ $C^{1,1}(\Omega)$ of a locally convex surface $\Sigma$ in terms of the first fundamental form $I_{\Sigma}$, the Gauss curvature $K$ and the integral $\int|H| d \sigma$. Here $H$ is the mean curvature of $\Sigma$. The coefficients $g_{i j}$ of $I_{\Sigma}$ are assumed to belong to the Hölder class $C^{2, \mu}(\Omega)$ for some $\mu, 0<\mu<1$. A boundary condition is discussed which ensures an estimate for $\int|H| d \sigma$.

\section{INTRODUCTION AND STATEMENT OF THE RESULTS}

Let $\Omega$ be a domain in the $u=\left(u^{1}, u^{2}\right)$-plane. Consider a differential geometric, locally convex surface $\Sigma$, which is given by a radius vector $X$ of class $C^{1,1}\left(\Omega, \mathrm{R}^{3}\right)$ such that the unit normal

$$
\nu=\frac{D_{1} X \wedge D_{2} X}{\left|D_{1} X \wedge D_{2} X\right|}
$$

exists.

AssumPtion (A). Suppose that the coefficients $g_{i j}$ of the first fundamental form

$$
\begin{aligned}
I_{\Sigma} & =D_{i} X \cdot D_{j} X d u^{i} d u^{j} \\
& =g_{i j} d u^{i} d u^{j}
\end{aligned}
$$

belong to the Hölder class $C^{2, \mu}(\Omega)$ for some $\mu, 0<\mu<1$, such that

and

$$
\left\|g_{i j}\right\|_{C^{2, \mu}(\Omega)} \leqslant a
$$

Here

$$
g, K \geqslant \frac{1}{c}
$$

and

$$
\begin{gathered}
g=\operatorname{det} I_{\Sigma}=\left|D_{1} X \wedge D_{2} X\right|^{2}, \\
K=\frac{\operatorname{det} I I_{\Sigma}}{\operatorname{det} I_{\Sigma}}=\frac{h}{g}
\end{gathered}
$$

Received 9 January 1990

Most of the research for this article was carried out in Fall 1988 when I visited the University of Kentucky in Lexington. I gratefully acknowledge the support by NSF grant RII-8610671 and the Commonwealth of Kentucky through the Kentucky EPSCoR Program. It is a pleasure to thank Professor R. Gariepy and all participants of the EPSCoR Seminar for their warm hospitality during my stay in Lexington.

Copyright Clearance Centre, Inc. Serial-fee code: 0004-9729/90 \$A2.00+0.00. 
is the Gauss curvature of $\Sigma$, which, by the theorema egregium, depends only on the coefficients of $I_{\Sigma}$ and their first and second derivatives.

$$
\begin{aligned}
I I_{\Sigma} & =D_{i j} X \cdot \nu d u^{i} d u^{j} \\
& =h_{i j} d u^{i} d u^{j}
\end{aligned}
$$

is the second fundamental form, which is defined almost everywhere.

Assumption(B). Suppose that

where

$$
\begin{gathered}
\int_{\Sigma}|H| d \sigma \leqslant M, \\
H=\frac{h_{i j} g^{i j}}{2}, \quad\left[g^{i j}\right]=\left[g_{k \ell}\right]^{-1},
\end{gathered}
$$

is the mean curvature, and

$$
d \sigma=\sqrt{g} d u
$$

is the area element of $\Sigma$.

The main result of this note then reads as the following:

THEOREM 1. The radius vector $X$ belongs to the Hölder class $C_{\text {loc }}^{2, \mu}(\Omega)$. For each subset $\Omega^{\prime}$, which is compactly contained in $\Omega$, there is an estimate of the form

$$
\left\|D^{2} X\right\|_{C^{\mu}\left(\Omega^{\prime}\right)} \leqslant C,
$$

where the constant $C$ only depends on $\mu, a, c, M$, and dist $\left(\Omega^{\prime}, \partial \Omega\right)$.

The regularity part of Theorem 1 follows from the regularity theory for elliptic Monge-Ampère equations [27] via the Darboux equation (5) (see for example, Nirenberg [18]). The $C_{\text {loc }}^{2, \mu}$-estimates follow from $[24,26]$, if $\Sigma$ is a graph or a closed surface. These results can also be derived from the prescribed Gauss curvature equation

$$
\operatorname{det} D^{2} z=K\left(1+|D z|^{2}\right)^{2}
$$

(compare Sabitov [20] for the regularity and $[\mathbf{2 2}, \mathbf{2 3}]$ for the a priori estimates for graphs and closed surfaces). The prescribed Gauss curvature equation (2) is particularly useful when the regularity requirements regarding $I_{\Sigma}$ are weakened to the extent that the Gauss curvature $K$ is only pinched between two positive numbers (see Heinz [6], Nikolaev and Shefel' $[16,17])$.

The regularity statement can be considered a variation of regularity theorems of Alexandrow [1] and Pogorelov [19]. That it is sharp follows from Sabitov and Shefel' [21], who investigated the connections between the regularity of a surface and its metric. 
The case of closed surfaces is of particular interest because of Weyl's embedding problem (see Weyl [29], Lewy [14], Nirenberg [18], Heinz [5, 22, 23]).

The purpose of the present note however is to provide the stated local $C^{2, \mu_{-}}$ estimates for the radius vector $X$ of a locally convex surface, thus improving those of Heinz [7], which require additional regularity assumptions regarding both the radius vector $X(u)$ and the coefficients $g_{i j}$ of the first fundamental form. The approach, which is due to Heinz [7], consists of introducing conjugate isothermal parameters, that is, of constructing a conformal map $x=x(u)$ with respect to the second fundamental form of $\Sigma$.

The present estimates rest on sharp estimates for the Jacobian of the Darboux system (8), which is satisfied by the inverse mapping $u=u(x)$. These estimates were derived in [25], generalising classical theorems of Lewy [12, 13] (see also Efimow [4]) and Heinz $[7,8]$.

The a priori constant in (1) does depend on the integral $\int|H| d \sigma$, because suitable Riemannian metrics on the unit disc with positive Gauss-Kronecker curvature can be embedded in Euclidean 3-space such that $\int|H| d \sigma$ is arbitrarily large (see Theorem 3 of Heinz [9]).

If $\Sigma$ is a closed convex surface, then the integral $\int|H| d \sigma$ can be estimated because of Minkowski's integral formula

$$
\int_{\Sigma} H d \sigma=-\int_{\Sigma} K \nu \cdot X d \sigma
$$

which holds for orientable closed surfaces (Minkowski [15], Herglotz. [10], Efimow [4], Heinz [7]). A careful investigation of the proof of (3), (which we take from Klingenberg [11], p.106, instead of proving (3) like in [7]) shows that an a priori estimate for $\int|H| d \sigma$ can also be derived if $\Sigma$ is attached to the unit sphere $S^{2}$ of order 1 :

Proposition 2. Suppose that $X \in C^{1,1}(B) \cap C_{l o c}^{2}(B), B=\{|u|<1\}$, is the radius vector of a locally convex surface, which satisfies the boundary condition

$$
|X|=1, \quad \frac{\partial|X|}{\partial n}=0 \text { for }|u|=1 .
$$

Here $n$ is the outward pointing normal to $\partial B=S^{1}$. Then there is an estimate of the form

where

$$
\begin{gathered}
\int_{\Sigma}|H| d \sigma \leqslant C(a, \kappa) \\
\left|g_{i j}\right| \leqslant a, \quad K \leqslant \kappa .
\end{gathered}
$$




\section{The Darboux equation and the Regularity proof}

Let

$$
\rho=\rho(u)=X \cdot X_{0}, \quad X_{0}=\nu\left(u_{0}\right),
$$

for some $u_{0} \in \Omega$. The Gauss equations

$$
\begin{gathered}
D_{i j} X=\Gamma_{i j}^{k} D_{k} X+h_{i j} \nu \\
\Gamma_{i j}^{k}=\frac{1}{2} g^{k \ell}\left(D_{j} g_{i \ell}+D_{i} g_{j \ell}-D_{\ell} g_{i j}\right)
\end{gathered}
$$

then imply that

$$
\begin{aligned}
\operatorname{det}\left[D_{i j} \rho-\Gamma_{i j}^{k} D_{k} \rho\right] & =h\left(\nu \cdot X_{0}\right)^{2} \\
& =K\left(D_{1} X D_{2} X X_{0}\right)^{2} \\
& =K\left[g-\frac{g^{i j}}{g} D_{i} \rho D_{j} \rho\right]
\end{aligned}
$$

(5) is the Darboux equation, which is elliptic in a neighbourhood $\mathcal{N}$ of $u_{0} \in \Omega$, because $\Sigma$ is then a convex graph over a plane perpendicular to $\nu\left(u_{0}\right)$. The regularity theory for elliptic Monge-Ampère equations, in particular Theorem 1 of $[\mathbf{2 7}]$, yields the regularity $\rho \in C_{\text {loc }}^{2, \mu}(\mathcal{N})$. To translate this into the regularity $X \in C_{\text {loc }}^{2, \mu}\left(\mathcal{N}, \mathbf{R}^{3}\right)$, consider the three $3 \times 3$-systems

$$
\begin{aligned}
X_{0} \cdot D_{i j} X & =D_{i j} \rho, \\
D_{k} X \cdot D_{i j} X & =\frac{1}{2}\left(D_{j} g_{i k}+D_{i} g_{j k}-D_{k} g_{i j}\right)
\end{aligned}
$$

(which can easily be derived from the Gauss equations). By (5), the determinant of the coefficient matrix is

$$
X_{0} D_{1} X D_{2} X=\sqrt{g-\frac{g^{i j}}{g} D_{i} \rho D_{j} \rho} \neq 0
$$

and the statement $X \in C^{2, \mu}\left(\Omega, \mathbf{R}^{3}\right)$ of Theorem 1 follows from Cramer's rule.

3. Conjugate isothermal parameters and the Darboux system

LEMMA 3. Let $a_{i j}$ be functions of class $C^{1}(\Omega)$ such that

$$
\Delta=\operatorname{det}\left[a_{i j}\right]>0 \text {. }
$$


Let $\bar{B}_{R}=\bar{B}_{R}\left(u_{0}\right) \subset \Omega$. Then there exists a homeomorphism $u=u(x)$ from $\bar{B}=$ $\{|x| \leqslant 1\}$ onto $\bar{B}_{R}$ of class $C_{\text {loc }}^{1, \mu}(B)$ with $u(0)=u_{0}$, which satisfies the system

$$
D_{\alpha}\left(\sqrt{\Delta} D_{\alpha} u^{k}\right)=D_{\alpha}\left(\Delta a^{\alpha k}\right) D u^{1} \wedge D u^{2} \text {. }
$$

Furthermore

$$
D u^{1} \wedge D u^{2}=D_{1} u^{1} D_{2} u^{2}-D_{2} u^{1} D_{1} u^{2} \neq 0 \text {, }
$$

and

$$
\sqrt{\Delta} a^{i j}=\frac{D u^{i} \cdot D u^{j}}{D u^{1} \wedge D u^{2}} \text {. }
$$

This lemma is contained in Lemma 2 of [26], which in turn is an improved version of Lemma 2 of Heinz [5]. The proof is by mapping the disc $\bar{B}_{R}\left(u_{0}\right)$ conformally with respect to the metric

$$
d s^{2}=a_{i j} d u^{i} d u^{j}
$$

onto the unit disc $\bar{B}=\{|x| \leqslant 1\}$.

PROPOSITION 4. Suppose that $\Sigma$ is a locally convex surface with radius vector $X \in C^{2, \mu}\left(\Omega, \mathbf{R}^{3}\right)$ for some $\mu, 0<\mu<1$, and let $\bar{B}_{R}=\bar{B}_{R}\left(u_{0}\right) \subset \Omega$. Then there exist conjugate isothermal parameters $x=\left(x^{1}, x^{2}\right)$, that is, there exists a homeomorphism $u=u(x)$ from $\bar{B}=\{|x| \leqslant 1\}$ onto $\bar{B}_{R}$ of class $C_{\text {loc }}^{1, \mu}(B)$ with $u(0)=u_{0}$, and

$$
D u^{1} \wedge D u^{2}=D_{1} u^{1} D_{2} u^{2}-D_{2} u^{1} D_{1} u^{2}>0
$$

such that the following conformality relations hold:

$$
\sqrt{g K} h^{i j}=\frac{D u^{i} \cdot D u^{j}}{D u^{1} \wedge D u^{2}} .
$$

Furthermore $u$ satisfies the Darboux system

$$
\Delta_{K} u^{k}=D_{\alpha}\left(\sqrt{K} D_{\alpha} u^{k}\right)+\sqrt{K} \Gamma_{i j}^{k} D u^{i} \cdot D u^{j}=0 \quad(k=1,2) .
$$

PRoof: Assume first that $X \in C^{3}\left(\Omega, \mathrm{R}^{3}\right)$ so that $I I_{\Sigma} \in C^{1}\left(\Omega, \mathrm{R}^{3}\right)$ and consider the differential form

$$
\begin{aligned}
d s^{2} & =\frac{1}{\sqrt{g}} I I_{\Sigma} \\
& =\frac{h_{i j}}{\sqrt{g}} d u^{i} d u^{j} .
\end{aligned}
$$

Lemma 3 yields the existence of the parameters $x=\left(x^{1}, x^{2}\right)$ which satisfy the conformality relations (7). According to (6),

that is,

$$
\begin{aligned}
& D_{\alpha}\left(\sqrt{K} D_{\alpha} u^{k}\right)=D_{\alpha}\left(\sqrt{g} K h^{\alpha k}\right) D u^{1} \wedge D u^{2}, \\
& D_{\alpha}\left(\sqrt{K} D_{\alpha} u^{1}\right)=\left[D_{1}\left[\frac{h_{22}}{\sqrt{g}}\right]-D_{2}\left[\frac{h_{12}}{\sqrt{g}}\right]\right] D u^{1} \wedge D u^{2}, \\
& D_{\alpha}\left(\sqrt{K} D_{\alpha} u^{2}\right)=\left[D_{2}\left[\frac{h_{11}}{\sqrt{g}}\right]-D_{1}\left[\frac{h_{12}}{\sqrt{g}}\right]\right] D u^{1} \wedge D u^{2} .
\end{aligned}
$$


By invoking the Codazzi-Mainardi equations

and

$$
\begin{aligned}
D_{j} h_{i k}-D_{i} h_{j k} & =\Gamma_{j k}^{\ell} h_{i \ell}-\Gamma_{i k}^{\ell} h_{j \ell} \\
D_{k} g & =2 g\left(\Gamma_{1 k}^{1}+\Gamma_{2 k}^{2}\right), \\
D_{k} g_{i j} & =\Gamma_{i k}^{\ell} g_{\ell j}+\Gamma_{j k}^{\ell} g_{\ell i},
\end{aligned}
$$

which follow from

one computes

$$
\begin{aligned}
D_{1}\left[\frac{h_{22}}{\sqrt{g}}\right]-D_{2}\left[\frac{h_{12}}{\sqrt{g}}\right] & =\frac{1}{\sqrt{g}}\left(\Gamma_{12}^{\ell} h_{2 \ell}-\Gamma_{22}^{\ell} h_{1 \ell}-h_{22}\left(\Gamma_{11}^{1}+\Gamma_{21}^{2}\right)+h_{12}\left(\Gamma_{12}^{1}+\Gamma_{22}^{2}\right)\right) \\
& =\frac{1}{\sqrt{g}}\left(-\Gamma_{11}^{1} h_{22}+2 \Gamma_{12}^{1} h_{12}-\Gamma_{22}^{1} h_{11}\right) \\
& =-\sqrt{K} \Gamma_{i j}^{1} \frac{D u^{i} \cdot D u^{j}}{D u^{1} \wedge D u^{2}}
\end{aligned}
$$

and

$$
\begin{aligned}
D_{2}\left[\frac{h_{11}}{\sqrt{g}}\right]-D_{1}\left[\frac{h_{12}}{\sqrt{g}}\right] & =\frac{1}{\sqrt{g}}\left(\Gamma_{21}^{\ell} h_{1 \ell}-\Gamma_{11}^{\ell} h_{2 \ell}-h_{11}\left(\Gamma_{12}^{1}+\Gamma_{22}^{2}\right)+h_{12}\left(\Gamma_{11}^{1}+\Gamma_{21}^{2}\right)\right) \\
& =\frac{1}{\sqrt{g}}\left(-\Gamma_{11}^{2} h_{22}+\Gamma_{21}^{2} h_{12}-\Gamma_{22}^{2} h_{11}\right) \\
& =-\sqrt{K} \Gamma_{i j}^{2} \frac{D u^{i} \cdot D u^{j}}{D u^{1} \wedge D u^{2}}
\end{aligned}
$$

The statement remains true if $X \in C^{2, \mu}\left(\Omega, \mathrm{R}^{3}\right)$. This is seen by essentially repeating the approximation argument in the proof of Lemma 2 of [26]: Let $\left\{X^{(n)}\right\}_{n=1}^{\infty}$ be $C^{3}\left(\Omega, \mathrm{R}^{3}\right)$-mappings which approximate the radius vector $X$ and its first and second derivatives uniformaly in $\bar{B}_{R}$. The regularity theory for linear equations (see [25]) yields local $C^{1, \mu}$-estimates for the approximating mappings $\left\{u^{(n)}\right\}_{n=1}^{\infty}$, because $K=h / g \in$ $C^{\mu}(\Omega)$, and since the conformality relations for $u^{(n)}$ imply that

$$
\begin{aligned}
\int_{B}\left|D u^{(n)}\right|^{2} d x & \leqslant C \int_{B} D u^{1} \wedge D u^{2} d x \\
& =C \iint_{B_{R}} d u \\
& =C R^{2} .
\end{aligned}
$$

Hence there exists a limit mapping $u=u(x)$, which is univalent because the inverses $x^{(n)}=x^{(n)}(u)$ are equicontinuous in $\bar{B}_{R}$ by the Courant-Lebesgue lemma. 
This is true because the conformality relations for $u^{(n)}$ also imply that

$$
\begin{aligned}
\int_{B_{R}}\left|D x^{(n)}\right|^{2} d u & \leqslant C \int_{B_{R}} D x^{1} \wedge D x^{2} d u \\
& =C \int_{B} d x \\
& =C .
\end{aligned}
$$

In order to conclude that $u=u(x)$ is a diffeomorphism from $B$ onto $B_{R}$, consider the integrability conditions for the inverses $x^{(n)}=x^{(n)}(u)$, the elliptic system

$$
D_{j}\left(h^{i j(n)} D_{i} x^{(n)}\right)=0,
$$

which has Hölder continuous coefficients. Then there are $C_{\text {loc }}^{1, \mu}$-estimates for $\left\{(x)^{(n)}\right\}_{n=1}^{\infty}$, and the limit mapping $x=x(u)$ is therefore of class $C_{\text {loc }}^{1, \mu}\left(B_{R}\right) \cap C^{0}\left(\bar{B}_{R}\right)$, which is the inverse of $u=u(x)$. This in turn implies the nonvanishing of $D u^{1} \wedge D u^{2}$ and the relations (7) are therefore satisfied.

\section{A PRIORI ESTIMATES FOR LOCALLY CONVEX SURFACES}

LemMa 5. Let $\Sigma$ be a locally convex surface with radius vector $X \in C^{2, \mu}\left(\Omega, \mathrm{R}^{3}\right)$ for some $\mu, 0<\mu<1$. Suppose that

$$
\begin{gathered}
\left|g_{i j}\right| \leqslant a, \\
g, K \geqslant \frac{1}{c} .
\end{gathered}
$$

Then the mapping $u=u(x), x \in B$, from Proposition 4, satisfies the estimate

$$
\int_{B}|D u|^{2} d x \leqslant C(a, c) \int_{\Sigma}|H| d \sigma .
$$

Proof: The mean curvature $H$ of $\Sigma$ can be estimated by the conformality relations (7):

$$
\begin{aligned}
|H| & =\left|\frac{h_{i j} g^{i j}}{2}\right| \\
& =\left|\frac{h}{2 g} g_{i j} h^{i j}\right| \\
& =\frac{1}{2} \sqrt{\frac{K}{g}} g_{i j} \frac{D u^{i} \cdot D u^{j}}{\left|D u^{1} \wedge D u^{2}\right|} \\
& \geqslant \frac{1}{2} \sqrt{\frac{K}{g}} \frac{g}{2 a} \frac{|D u|^{2}}{\left|D u^{1} \wedge D u^{2}\right|} \\
& \geqslant \frac{1}{4 a c} \frac{|D u|^{2}}{\left|D u^{1} \wedge D u^{2}\right|} .
\end{aligned}
$$


Therefore

$$
\begin{aligned}
\int_{B}|D u|^{2} d x & \leqslant 4 a c \int_{B_{R}\left(u_{0}\right)}|H| d u \\
& \leqslant 4 a \sqrt{c} \int_{\Sigma}|H| d \sigma .
\end{aligned}
$$

Proof of Theorem 1 (of the a priori estimates): Let $\bar{B}_{R}=\bar{B}_{R}\left(u_{0}\right) \subset \Omega$. Consider the homeomorphism $u=u(x)$ from $\bar{B}$ onto $\bar{B}_{R}$ from Proposition 4. Now $u$ is of class $C_{\text {loc }}^{1, \mu}(B)$ and its Dirichlet integral is estimated by (9). Furthermore

$$
\begin{aligned}
\sqrt{g} K h^{i j} & =\frac{D u^{i} \cdot D u^{j}}{D u^{1} \wedge D u^{2}} \\
\Delta_{K} u & =0
\end{aligned}
$$

Suppose now, without loss of generality, that $B_{R}\left(u_{0}\right)=B=\{|u|<1\}$ (otherwise consider the mapping $\left.\frac{1}{R} u(x)-u_{0}\right)$. The Main Theorem of [25] can then be applied to the system (11) to give the following estimates in any disc $B_{\rho}=\{|x|<\rho\}, 0<\rho<1$ :

$$
\begin{aligned}
\|u\|_{C^{1, \mu}\left(B_{\rho}\right)} & \leqslant C(\ldots, \rho), \\
D u^{1} \wedge D u^{2} & \geqslant c(\ldots, \rho)>0 .
\end{aligned}
$$

By taking $\rho=1 / 2$, and then also taking into account that we assumed that $B_{R}\left(u_{0}\right)=$ $B$, the relations (10) yield the bounds

$$
\left|h_{i j}\left(u_{0}\right)\right| \leqslant C(\ldots, R)
$$

from which, in $\Omega^{\prime}$,

$$
\left|h_{i j}\right| \leqslant C\left(\ldots, \operatorname{dist}\left(\Omega^{\prime}, \partial \Omega\right)\right) .
$$

Furthermore the functions $h_{i j}(u(x))$ satisfy Hölder estimates of the form

$$
\left[h_{i j}\right]_{\mu}^{B_{\rho}} \leqslant C(\ldots, \rho, R)
$$

in each $B_{\rho}=\{|x|<\rho\}$. In order to translate this into estimates for $h_{i j}(u)$, note that the estimate (9) for the Dirichlet integral of $u$ implies, by the Courant Lebesgue lemma, that there exists a $\rho=\rho(a, c, \mu, R), 0<\rho<1$, such that $x \in B_{\rho}$ if $u \in B_{R / 2}\left(u_{0}\right)$. Since

$$
x_{k}=\int_{0}^{1} D x_{k}\left(u_{0}+\tau\left(u-u_{0}\right)\right) \cdot\left(u-u_{0}\right) d \tau
$$


the estimates $(12,13)$ yield a dilation inequality of the form

$$
|x| \leqslant C(\ldots, R)\left|u-u_{0}\right|
$$

if $u \in B_{R / 2}\left(u_{0}\right)$, and therefore

$$
\left|h_{i j}(u)-h_{i j}\left(u_{0}\right)\right| \leqslant C(\ldots, R)\left|u-u_{0}\right|^{\mu}
$$

which implies the Hölder estimates

$$
\left[h_{i j}\right]_{\mu}^{\Omega^{\prime}} \leqslant C\left(\ldots, \operatorname{dist}\left(\Omega^{\prime}, \partial \Omega\right)\right) .
$$

A priori estimates for the second derivatives of the radius vector $X$ follow from the Gauss equations as required.

\section{Proof of Proposition 2}

If $X \in C^{3}(B)$, then

$$
D_{i}\left(\sqrt{g} K h^{i j} D_{j} X\right)=2 \sqrt{g} K \nu
$$

This formula is easily shown to hold in Fermi coordinates (see [11], pp.104, 106). By dotting with $X$ and integrating over $B_{\rho}, 0<\rho<1$, it follows that

$$
\begin{aligned}
2 \int_{\Sigma_{\rho}} K \nu \cdot X d \sigma & =-\int_{B_{\rho}} \sqrt{g} K g_{i j} h^{i j} d u+\frac{1}{2} \int_{\partial B_{\rho}} \sqrt{g} K h^{i j} D_{j}|X|^{2} n_{i} d s \\
& =-2 \int_{\Sigma_{\rho}} B d \sigma+\frac{1}{2} \int_{\theta B_{\rho}} \sqrt{g} K h^{i j} D_{j}|X|^{2} n_{i} d s .
\end{aligned}
$$

This relation holds true if $X \in C^{2}(B)$. By letting $\rho \rightarrow 1$ and incorporating the boundary condition (4), it follows that

$$
\int_{\Sigma} K \nu \cdot X d \sigma=-\int_{\Sigma} H d \sigma
$$

Finally, $K>0$ implies the required estimate

$$
\begin{aligned}
\int_{\Sigma}|H| d \sigma & \leqslant C(a, \kappa)|X| \\
& \leqslant C(a, \kappa) .
\end{aligned}
$$




\section{REFERENCES}

[1] A.D. Alexandrow, Die innere Geometrie der konvexen Flächen (Akademie-Verlag, Berlin, 1955).

[2] Ph. Delanoë, 'Réalisations globalement régulières de disques strictement convexes dans les espaces d'Euclide et de Minkowski par la méthode de Weingarten', Ann. Sci. École Norm. Sup. (4) 21 (1988), 637-652.

[3] D.M. Deturck and J.L. Kazdan, 'Some regularity theorems in Riemannian geometry', Ann. Sci. École. Norm. Sup. (4) 14 (1981), 249-260.

[4] N.M. Efimow, Flächenverbiegungen im Grossen, (mit einem Nachtrag von E. Rembs und K.P. Grotemeyer) (Akademie-Verlag, Berlin, 1957).

[5] E. Heinz, 'On elliptic Monge-Ampère equations and Weyl's embedding problem', $J$. Analyse Math. 7 (1959), 1-52.

[6] E. Heinz, 'Über die Differentialungleichung $0<\alpha \leqslant r t-s^{2} \leqslant \beta<\infty$ ', Math. Z. 72 (1959), 107-126.

[7] E. Heinz, 'Neue a-priori-Abschätzungen für den Ortsvektor einer Fläche positiver Gaussscher Krümmung durch ihr Linienelement', Math. Z. 74 (1960), 129-157.

[8] E. Heinz, 'Über das Nichtverschwinden der Funktionaldeterminante bei einer Klasse eineindeutiger Abbildungen', Math. Z. 105 (1968), 87-89.

[9] E. Heinz, 'Existence theorems for one-to-one mappings associated with elliptic systems of second order II', J. Analyse Math. 17 (1966), 145-184.

[10] G. Herglotz, 'Über die Starrheit der Eiflächen', Abh. Math. Sem. Univ. Hamburg 15 (1943), 127-129.

[11] W. Klingenberg, Eine Vorlesung über Differentialgeometrie (Springer-Verlag, Berlin, Heidelberg, New York, 1973).

[12] H. Lewy, 'On the non-vanishing of the Jacobian in certain one-to-one mappings', $B$ ull. Amer. Math. Soc. 42 (1936), 689-692.

[13] H. Lewy, 'A priori limitations for solutions of Monge-Ampère equations', Trans. Amer. Math. Soc. 37 (1935), 417-434.

[14] H. Lewy, 'On the existence of a closed convex surface realizing a given Riemannian metric', Proc. Nat. Acad. Sci. U.S.A. 24 (1938), 104-106.

[15] H. Minkowski, 'Volumen und Oberfläche', Math. Ann. 57 (1903), 447-495.

[16] I.G. Nikolaev and S.Z. shefel', 'Smoothness of convex surfaces on the basis of differential properties of quasiconformal mappings', Soviet Math. Dokl. 26 (1982), 599-602.

[17] I.G. Nikolaev and S.Z. Shefel', 'Convex surfaces with positive bounded specific curvature and a priori estimates for Monge-Ampère equations', Siberian Math. J. 26 (1985), 572-586.

[18] L. Nirenberg, 'The Weyl and Minkowski problems in differential geometry in the large', Comm. Pure Appl. Math. 6 (1953), 337-394.

[19] A.V. Pogorelov, Extrinsic geometry of convex surfaces (American Mathematical Society, Providence, Rhode Island, 1973).

[20] I. Kh. Sabitov, "The regularity of convex regions with a metric that is regular in the 
Hölder classes', Siberian Math. J. 17 (1976), 681-687.

[21] I. Kh. Sabitov and S.Z. Shefel', 'The connections between the order of smoothness of a surface and its metric', Siberian Math. J. 17 (1976), 687-694.

[22] F. Schulz, 'On elliptic Monge-Ampère equations with a remark on Weyl's embedding problem', Nachr. Akad. Wiss. Göttingen Math.-Phys. Kl. II 1981 (1981), 93-108.

[23] F. Schulz, 'On the differential equation $r t-s^{2}=f$ and Weyl's embedding problem', Math. Z. 179 (1982), 1-10.

[24] F. Schulz, 'A priori estimates for solutions of.Monge-Ampère equations', Arch. Rational Mech. Anal. 89 (1985), 123-133.

[25] F. Schulz, 'Univalent solutions of elliptic systems of Heinz-Lewy type', Ann. Inst. H. Poincaré, Anal. Non Linéaire 6 (1989), 347-361.

[26] F. Schulz, 'Second derivative estimates for solutions of two-dimensional Monge-Ampère equations', Proc. Amer. Math. Soc. (to appear).

[27] F. Schulz and L.-Y. Liao, 'Regularity of solutions of two-dimensional Monge-Ampère equations', Trans. Amer. Math. Soc. 307 (1988), 271-277.

[28] S.Z. Shefel', 'Geometric properties of immersed manifolds', Siberian Math. J. 26 (1985), 133-147.

[29] H. Weyl, 'Über die Bestimmung einer geschlossenen Fläche durch ihr Linienelement', in Selecta Hermann Weyl, pp. 148-178 (Basel-Stuttgart, 1956).

Department of Mathematics

The University of Iowa

Iowa City, IA 52242

United States of America

and

Centre for Mathematical Analysis

The Australian National University

GPO Box 4

Canberra ACT 2601

Australia 